\title{
COMPUTING CAPABILITIES OF CRYSTALS
}

\author{
Aleksey A. Demidov ${ }^{1 *}$ \\ *1 Program Systems Institute of RAS, Russia \\ alex@dem.botik.ru
}

*Corresponding Author: -

EmailID-alex@dem.botik.ru

\begin{abstract}
: -
The work is devoted to investigation from the standpoint of quantum physics the possibility of using the scattering of a beam of light on crystals for calculations. There is proposed a model of computation, corresponding the transformation of the light beam as it passes through the crystal, and provided the necessary formulas for the calculation of the model. It is necessary to emphasize the abstract nature of the research, which is aimed at creation of the theoretical basis for further study of the produced algebraic structures, rather than the construction of a real device - a quantum computer or the like. The work carried out by the program of creation of algebraic computer [1].
\end{abstract}

Keywords: - parallel computing, program algebras, quantum optics, computation basics, crystals.

\section{(c) (\$) (1)}


Transforming a light beam by a crystal is usually associated with the scattering problem, which consists of determining the change in the spatial distribution, frequency, polarization of optical radiation and its interaction with matter [2,3]. A consistent solution of the scattering problem is only possible in the appropriate quantum theory - quantum electrodynamics. The elementary act of scattering is treated as an incident photon absorption by matter, and then the spontaneous emission of photons scattered from the different values of energy, momentum and polarization $[4,5]$. In this paper, scattering problem is considered simplistically: as we study the potential computing power of physical processes, the details of the conversion of the light beam by the crystal are ignored.

Let there be a crystal or an aggregation of crystals, which is supplied with the incoming light beam. Crystal transforms the beam in a unitary manner, after that the parameters of the outgoing beam are evaluated by a measuring device. Field vector $\mathbf{E}$ of a monochromatic wave could be written as

$$
\mathbf{E}=A e \mathbf{e}^{i(k r-o t)}
$$

where $A$ - amplitude, ${ }^{\mathbf{e}}$ - polarization vector, $\mathbf{k}$ - wave vector, ${ }^{\omega}$ - angular frequency [6][§ 1.2.1]. Any polarization vector ${ }^{\mathbf{e}}$ could be written as a decomposition of two orthogonal vectors, for example ${ }^{\mathbf{e}} \mathbf{x}$ and ${ }^{\mathbf{e}} \mathbf{y}$

$$
\mathbf{e}=a_{1} \mathbf{e}_{\mathbf{x}}+a_{2} e^{i \delta} \mathbf{e}_{\mathbf{y}}
$$

where $a^{1}=\cos \beta$ and $a_{2}=\sin \beta$ - real coefficients, $\beta$ - parameter (on linear polarization it could be taken as an angle between ${ }^{\mathbf{e}}{ }^{x}$ axis).

To describe the transformations of a complex beam, consisting of a plurality of rays, it is advisable to use the approach of quantum mechanics. In addition it provides the completeness of research of crystal capabilities. After that, the result could be roughened to the classic approach. It is possible to correspond a quantum state vector to each incoming ray

$$
|e\rangle=a_{1}\left|e_{x}\right\rangle+a_{2} e^{i \delta}\left|e_{y}\right\rangle
$$

The quantum state of complete system could be gained by the tensor products

$$
\psi=e_{1} \otimes \ldots \otimes \ddot{e}_{n}=\left|e_{1}\right\rangle \ldots\left|e_{n}\right\rangle
$$

The resulting state of the system could not be in general decomposed by the state of subsystems as in the formula (4), because because of non-separability. Therefore one often uses a density functional (matrix) ${ }^{\rho_{0}}$ [7], which is defined by the expression of non-separability. Therefore one often uses a density functional (matrix)

$$
\rho_{0}=\psi \psi^{*}=|\psi\rangle\langle\psi|,
$$

where the asterisk denotes a complex conjugation.

To evaluate the polarization properties of each ray, it is necessary to make four independent measurements, for example: evaluate Stokes parameters - intensity and three degrees of ray polarisation [6][\$ 1.2.5.4]. These parameters are used as incomming data to define the density matrix of the ray.

Denoting by $U$ the unitary transformation given by the crystal of a certain configuration at a certain position, one can write the resulting matrix

$$
\rho=U|\psi\rangle\langle\psi| U^{*}
$$

States of outgoing rays are defined by reduced density matrices, which could be obtained from $\rho$ by taken of a partial trace

$$
\begin{aligned}
& \rho^{(a)}=\operatorname{tr}_{B} \rho=\sum_{i}\left\langle B_{i}|\rho| B_{i}\right\rangle, \\
& \left\langle a_{j}\left|\rho^{(a)}\right| a_{k}\right\rangle=\sum_{i}\left\langle B_{i} a_{j}|\rho| B_{i} a_{k}\right\rangle,
\end{aligned}
$$

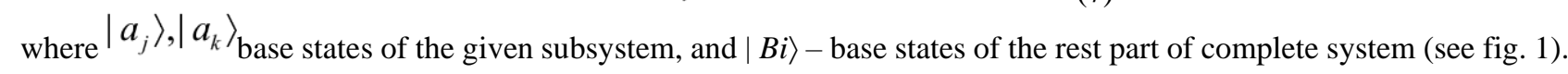

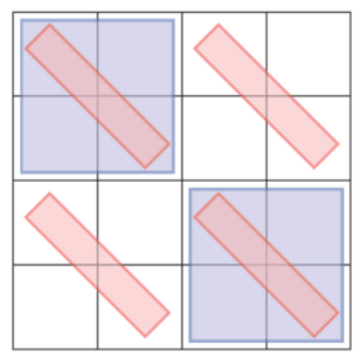

Fig 1. Operation of taking the partial trace. Elements of adjacent areas of the same color are summed in pairs. Operation $\rho^{(a)}=t r_{B} \rho$ corresponds to red colour, and $\rho^{(b)}=t r_{A} \rho$ to blue color. 
States (7) will in general be mixed, that is they will not be represented as a tensor product of vectors (5). In the incoherent mixture there are in a various proportion presented the components with the different state vectors, and such a decomposition of the mixed state in the pure states is not unique. The last fact is not so important, because different beams will behave identically in all the experiments in sense of the polarization properties if they are described by the the same density matrices [6] [\$ 1.1.5.3]. We will use a uniqueness of spectral decomposition of the matrix in the basis of eigenvectors

$$
\rho^{(n)}=V \Lambda V^{-1}
$$

where $V$ - matrix of eigenvectors of matrix $\rho^{(\mathrm{n})}$ by columns, ${ }^{\Lambda}{ }_{-}$diagonal matrix of eigenvalues of matrix ${ }^{\rho(n)}$ by main diagonal. Then a ray $n$ could be described as a set of the eigenvectors taken with statistic weights, which are represented by the eigenvalues:

$$
O^{(n)}=\bigcup_{i} \Lambda_{i i}\left|V_{i}\right\rangle
$$

Operation of specrtal decomposition is a complicated task in dimension $\left|\rho^{(n)}\right|>3$, because the power of algebraic equation which is arises on searching the eigenvalues of matrix equation

$$
\left(\rho^{(n)}-\lambda I\right) \mathbf{v}=0, \quad \operatorname{det}\left(\rho^{(n)}-\lambda I\right)=0,
$$

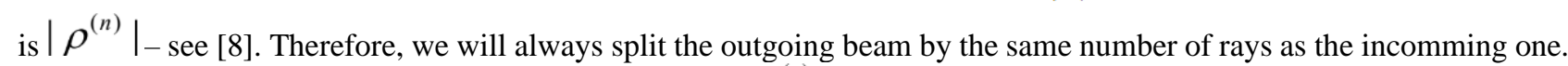
Then the dimensionality of density matrix of each ray is $\left|\rho^{(n)}\right|=2$.

Computational model, which corresponds of transformations implemented by the crystal, will depend on what objects will be taken as an elements: it is possible to get the density matrix of the complete system, not to measure individual rays - formula (6), or to be interested in the state of each incoming and outgoing ray - formula (9). The first option is only valid for the case of the cascade connection of crystals, and the final operation in any case should be measuring the state of each ray.

\section{REFERENCES}

[1].Nepeivoda N. N. From the numerical simulation to the algebraic one // Proceedings of the VI International Conference "Parallel Computing and Control Problems" (PACO-2012). - V. A. Trapeznikov Institute of Control of RAS 24. Vol. 1, pp. 93-103.

[2].Udem, Th., Holzwarth, R., Hänsch, T. W. Optical frequency metrology. Nature 416 (6877), 2002, pp. $233-237$.

[3].Physical encyclopedia. In 5 volumes. - Soviet encyclopedia. Editor in Chief Prokhorov A.M., 1988.

[4].Landau L.D., Lifshitz E.M. Course of Theoretical Physics. In 10 volumes. Vol. IV / Berestetski V.B., Lifshitz E.M., Pitaevskii L.P. Quantum electrodynamics. - Nauka, 1989. - 728 p.

[5].Fickler R., Lapkiewicz R., Plick W. N., Krenn M., Schaeff C., Ramelow S., Zeilinger A. Quantum entanglement of high angular momenta, Science 338, 2012, pp. 640-643.

[6].Blum K. Density Matrix Theory and Applications. 2nd ed. - Springer, 1996. - 323 p.

[7].Nikitin N.V. Density Matrix. Lecture course. - Physics Department of M.V. Lomonosov Moscow State University, 2015. $-250 \mathrm{p}$.

[8].Wilkinson J.H. Algebraic Eigenvalue Problem. - Clarendon Press, 1988. - 662 p. 